Research Paper

\title{
Epithelial-mesenchymal transition induced by GRO- $\alpha$-CXCR2 promotes bladder cancer recurrence after intravesical chemotherapy
}

\author{
Lu Chen ${ }^{1, *}$, Xiu-Wu Pan 2,3,*, Hai Huang ${ }^{3, *}$, Yi Gao ${ }^{3}$, Qi-Wei Yang ${ }^{2,3}$, Lin-Hui Wang ${ }^{3}$, \\ Xin-Gang $\mathrm{Cu}^{2}$ and Dan-Feng $\mathrm{Xu}^{1}$ \\ ${ }^{1}$ Department of Urinary Surgery of Ruijin Hospital, Shanghai Jiaotong University, Shanghai 200025, China \\ ${ }^{2}$ Department of Urinary Surgery of Third Affiliated Hospital, Second Military Medical University, Shanghai 201805, China \\ ${ }^{3}$ Department of Urinary Surgery of Changzheng Hospital, Second Military Medical University, Shanghai 200003, China \\ *These authors have contributed equally to this work \\ Correspondence to: Xin-Gang Cui, email: xingangcui@126.com \\ Dan-Feng XU, email: nycnjc@126.com
}

Keywords: GRO- $\alpha$, CXCR2, bladder cancer, epithelial-mesenchymal transition, recurrence

Received: March 24, 2016

Accepted: March 17, 2017

Published: April 03, 2017

Copyright: Chen et al. This is an open-access article distributed under the terms of the Creative Commons Attribution License 3.0 (CC BY 3.0), which permits unrestricted use, distribution, and reproduction in any medium, provided the original author and source are credited.

\section{ABSTRACT}

Non-muscle invasive bladder cancers (NMIBC) are typically treated by transurethral resection with intravesical chemotherapy. However, the post-therapeutic incidence of tumor recurrence and progression to muscle invasive disease is high, and the underlying mechanism(s) remains unknown. In this study, we observed that recurrent bladder cancer cells exhibit a mesenchymal phenotype, which is initiated by the autocrine GRO-a signaling. Mechanically, the chemotherapeutic drug epidoxorubicin induces GRO-a expression in primary bladder cancer cells at G1/S phase via p38-dependent activation of NF-KB. GRO-a phosphorylation of Snail on Ser246 supports Snail's accumulation in the nucleus, and thereby promotes transcription repression activity of Snail from E-cadherin promoters. In accordance, disrupting the GRO-a-Snail axis in NMIBC represents a promising alternative to prevent post-therapeutic tumor progression and recurrence.

\section{INTRODUCTION}

Approximately $75-85 \%$ cases of newly diagnosed bladder cancer are non-muscle invasive bladder cancer (NMIBC), including non-muscle invasive papillary or flat lesions (pTa and pTis, respectively) and invasive urothelial carcinomas involving the lamina propria (pT1) [1]. NMIBC is typically treated by transurethral resection and intravesical chemotherapy. The American Urological Association (AUA) considers all high grade $\mathrm{pTa}, \mathrm{pTis}$ and $\mathrm{pT} 1$ to be at high risk for recurrence and progression; pT1 tumors in particular show a highly divergent behavior, with progression rates of $17 \%$ to $50 \%$, despite therapy. Muscle-invasive bladder carcinoma is clinically unfavorable, with a 5-year overall survival rate of $48 \%$ to $67 \%[2,3]$. Therefore, development of novel therapies is needed to maximize the benefits of treatment, notably reducing disease recurrence and preventing progression.

Tumor cells progress from non-invasive to malignant phenotypes via a series of critical steps that involve morphological changes referred to as the epithelialmesenchymal transition (EMT). EMT is a process originally observed during the embryonic development, in which cells lose epithelial characteristics [4, 5]. Activation of the latent EMT program confers upon cancer cells a distinct advantage for local invasion [6,7]. In deed, Baumgart et al. characterized the expression of E-cadherin, $\beta$-catenin, plakoglobin, and vimentin in a series of primary bladder tumors. Downregulation of epithelial markers was associated with disease progression in terms of both grade and stage (i.e., superficial vs. muscle-invasive cancer), and in univariate analyses, downregulation of either $\beta$-catenin or plakoglobin was associated with shorter disease-specific 
survival [8]. With respect to the chemotherapy, previous studies have demonstrated a close link between EMT and treatment of chemotherapeutic agents. For example, Li et al. previously showed that transient adriamycin treatment induced EMT and apoptosis simultaneously in breast cancer cells. Only the cells undergoing EMT showed enhanced invasion/metastasis and multidrug resistance [9]. In addition, Kajiyama et al. identified an association between chronic paclitaxel resistance and induction of EMT in epithelial ovarian carcinomas [10]. Based on these observations, we hypothesize that EMT may contribute to the malignant phenotypes of bladder cancers after receiving chemotherapy.

In the current study, we demonstrated that recurrent bladder cancer cells undergoing chemotherapy preferentially exhibited a mesenchymal phenotype. In particular, recurrent bladder cancer cells undergoing chemotherapy secret growth-regulated protein alpha (GRO- $\alpha$ ), which triggers the process of EMT through regulating phosphorylation and functions of Snail. In clinical settings, treatment of relapsed tumor using the regimen that resulted in remission of the primary tumor is suboptimal. The current study raises the possibility of preventing/delaying tumor relapse via manipulating specific signaling pathway(s).

\section{RESULTS}

\section{Recurrent bladder cancer cells exhibit a mesenchymal phenotype}

We obtained bladder cancer cells from three paired primary and relapsed tumor samples (PB1-3 vs. RB1-3). Routine morphologic observation showed that primary tumor cells grew in the form of tightly-packed colonies characteristic of epithelial cells, while most recurrent tumor cells looked flattened, spread actively and underwent cytoskeletal rearrangement (Figure 1A). These phenomena were associated with decreased expression of E-cadherin, formation of actin stress fibers (F-actin), and up-regulation of N-cadherin (Figure 1A and Supplementary Figure 1A). Compared with their corresponding primary counterparts, the invasion rate of the recurrent tumor cells increased by 5.7-8.2 fold (Figure 1B), without showing any change in cell proliferation(Supplementary Figure 1B).

EMT is a process reminiscent of CSCs. Within 7 days, all individual cells produced non-adherent multicellular oncospheres highly expressing bladder CSC markers, such as CD44 and 67LR (Supplementary Figure 1C). Noticeably, the recurrent bladder cancer cells showed a 2.1-5.8-fold increase in oncosphere-forming
A.

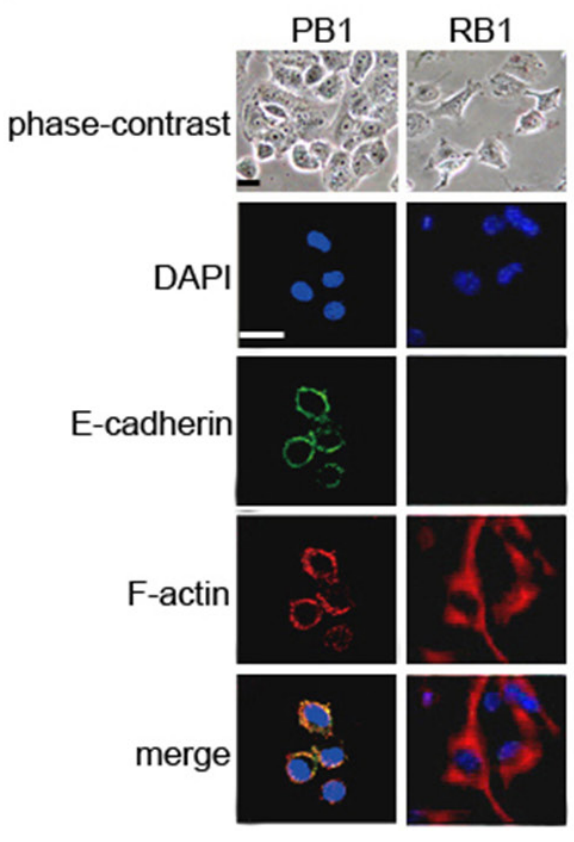

B.

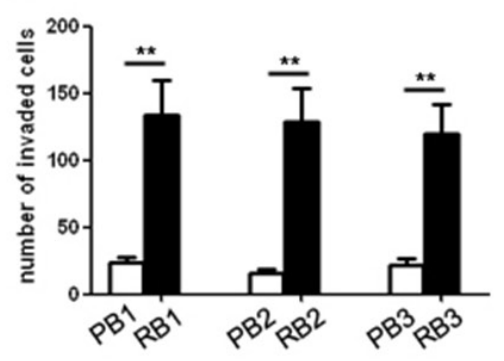

C.

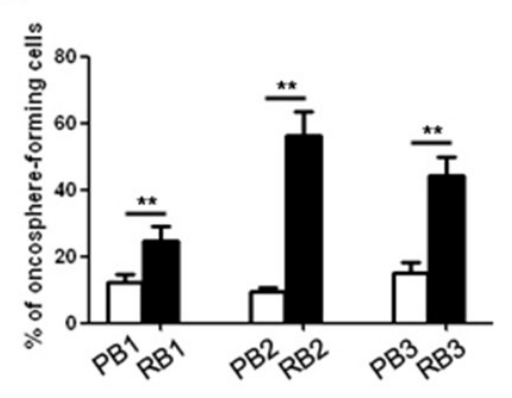

D.
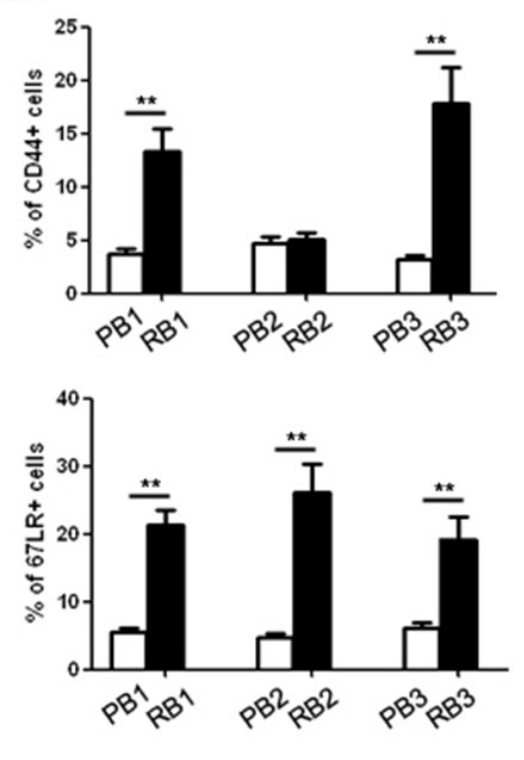

Figure 1: Recurrent bladder cancer cells exhibit a mesenchymal phenotype. (A) Morphology examination and immunofluorescence of E-cadherin, F-actin in paired primary and relapsed bladder cancer cells. (B) Invasive rate of the paired primary and relapsed bladder cancer cells. (C) Dissociated cells from the indicated tumor samples were incubated in the presence of growth factors for 7 days. The percentage of oncosphere-forming cells was determined. (D) The percentage of CD44- and 67LRe-positive cells were examined in the paired primary and recurrent bladder carcinomas by flow cytometric analysis. Experiments were performed three times independently, and results are shown as mean $\pm \mathrm{SD}$. ${ }^{* *}, \mathrm{p}<0.05$ versus the corresponding controls. Bars: $10 \mu \mathrm{m}$. 
capacity over the matched primary tumor cells (Figure 1C). Moreover, we performed limiting dilution assays to determine self-renewal capacity. Oncospheres from primary tumors showed limited expansion and could not be maintained beyond passage 2 ( $<2$ passages), while all recurrent tumors yielded sphere cells with extended passages ( $>4$ passages). To extend these observations, we examined the expression of putative CSC markers in the paired primary and recurrent bladder carcinomas by flow cytometric analysis. As compared with the matched primary tumors, most recurrent bladder cancer cells (RB1 and RB3) possessed a higher percentage of CD44- and 67LR-positive populations. Although there was only a negligible difference in the amount of CD44-positive cells between PB2 and RB2, RB3 possessed a 2.3-fold increase in 67LR-positive population compared with PB3 (Figure 1D). These data suggest that recurrent bladder cancer cells underwent epithelial-mesenchymal transition accompanied with malignant progression.

\section{GRO- $\alpha$ derived from recurrent bladder cancer cells elicits epithelial-mesenchymal transition}

To figure out whether the differential phenotypes were attributed to cancer cells themselves or the unique microenvironment, we isolated epithelial and stromal cells from recurrent tumor tissues and transferred them to the upper chamber of the Transwell, while the bottom compartment was seeded with the matched primary tumor cells (Figure 2A). This transwell system allows for exchange of diffusible factors but not cells between the chambers. PB1 cells cocultured with RB1 cells showed a fibroblast-like cell morphology. Furthermore, coculture with RB1 cells induced the loss of E-cadherin expression and formation of actin stress fibers in PB1 cells (Figure 2B). Correspondingly, increased expression of $\mathrm{N}$-cadherin and down-regulation of E-cadherin were also observed in PB1 cells (Supplementary Figure 2A). In contrast, no similar phenomena were observed in PB1 cells cocultured with stromal cells (Figure 2B and Supplementary Figure 2A), suggesting that recurrent bladder cancer cells themselves created a distinct environment for induction of EMT.

Using SINQ and MaxQuant label-free proteomic software, we performed a comparative analysis on differentially expressed proteins in the supernatant of recurrent bladder cancer cells. As compared with PB1 cells, 23 proteins in RB1 cells underwent differential expression by at least 3 fold, among which GRO- $\alpha$ underwent more than five-fold change. We further validated GRO- $\alpha$ expression, and found that GRO- $\alpha$ secretion, as well as its expression, in the recurrent cancer cells was significantly elevated as compared with that in their primary counterparts (Figure 2C). CXCR2 is the specific receptor for GRO- $\alpha$. Recurrent bladder cancer cells exhibited similar levels of CXCR2 in comparison with the corresponding primary cancer cells (Supplementary Figure 2B). These data suggest the existence of autocrine GRO- $\alpha$-CXCR2 signaling in recurrent bladder cancer cells.

Next, we evaluated the effect of GRO- $\alpha$ on EMT in bladder cancer cells. Following GRO- $\alpha$ treatment for $24 \mathrm{~h}$, primary PB2 cells exhibited a marked alteration in cell morphology, changing from the characteristic organized 'cobblestone' appearance of epithelial cell monolayers to a disorganized elongated fibroblastlike phenotype. In contrast, these cells treated with the specific CXCR2 inhibitor, SB-656933, in the presence of GRO- $\alpha$ maintained their epithelial phenotype (Figure 2D). Moreover, treatment of RB2 cells with SB-656933 led to morphological changes consistent with transition from a mesenchymal to an epithelial phenotype (Figure 2F). Consistent with the morphological findings, immunoblotting analysis of primary PB2 revealed diminished levels of E-cadherin and increased $\mathrm{N}$-cadherin expression following GRO- $\alpha$ treatment. In PB2 cells treated with SB-656933 and GRO- $\alpha$, the expression of both E-cadherin and vimentin remained unchanged, as compared with the control cells treated with the vehicle for both conditions (Figure 2E). As anticipated, in RB2 cells, inhibition of GRO- $\alpha$-CXCR2 signaling by SB-656933 potently upregulated the expression level of E-cadherin, while downregulated the mesenchymal marker N-cadherin (Figure 2G). These data suggest that mesenchymal transformation of recurrent bladder cancer cells was dependent on the autocrine GRO- $\alpha$-CXCR2 signaling.

\section{Epidoxorubicin induces GRO- $\alpha$ expression in bladder cancer cells in a cell cycle-dependent manner via the $\mathbf{p 3 8 / M A P K - N F - \kappa B}$ pathway}

The bladder cancer patients in our series experienced recurrence after intravesical chemotherapy. Epidoxorubicin, the drug used most often for intravesical chemotherapy, induced concurrence of different events (apoptosis vs. GRO- $\alpha$ secretion) in PB2 and PB3 cells (Figure 3A). To investigate the underlying mechanism, we examined the contribution of cell cycle state. PB2 and PB3 cells were synchronized at either G1/S or G2/M phase, after which epidoxorubicin-induced effects were examined. As shown in Figure 3B, epidoxorubicin induced GRO- $\alpha$ expression only in cells synchronized at $\mathrm{G} 1 / \mathrm{S}$ phase, whereas it induce apoptosis in cells synchronized at G2/M phase.

We then investigated potential transcription factors involved in regulation of GRO- $\alpha$ expression. The GRO- $\alpha$ promoter 5 '-flanking region (spanning from bp -980 to +76 ) and its sequentially deleted fragments (starting from $-980,-390,-100$ and -80 to $+76 \mathrm{bp}$ ) were prepared by PCR and cloned into luciferase expression vectors (Figure 3C). Stimulation with epidoxorubicin markedly increased the luciferase activity in constructs containing sequences from -980 to $-80 \mathrm{bp}$, which included NF- $\mathrm{KB}$ binding 
sites. However, the construct from which the sequence from -980 to $-80 \mathrm{bp}$ was deleted produced no response to drug treatment. The construct lacking the sequence from -980 to $-100 \mathrm{bp}$ produced the strongest luciferase activity in drugs-stimulated cells (Figure 3C), suggesting the involvement of NF- $\kappa \mathrm{B}$ in regulating GRO- $\alpha$ expression. To test this possibility, we evaluated the GRO- $\alpha$ promoter activity after cotransfection of the candidate transfactor in primary bladder cancer cells. It was found that the expression of p65 S276D (a constitutively active form of $\mathrm{NF}-\kappa \mathrm{B}$ ) was associated with a significant increase

A.

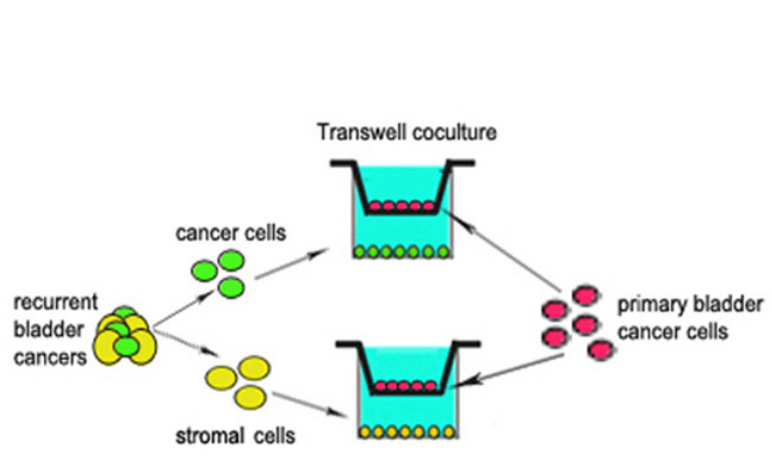

B.

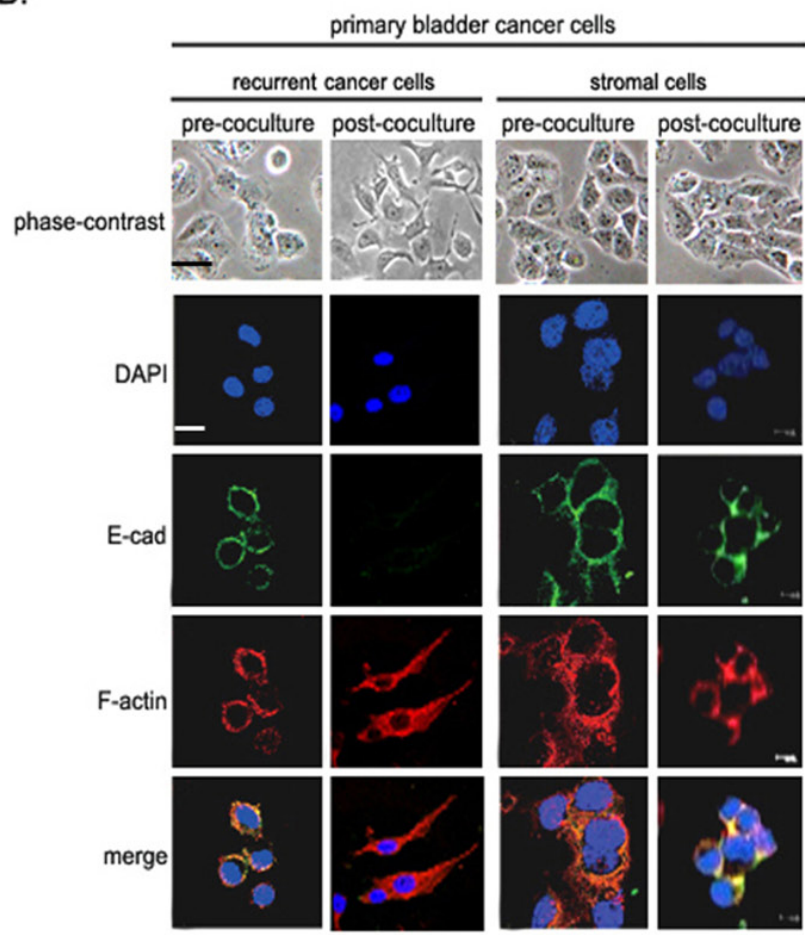

A.

. in the GRO- $\alpha$ promoter activity, while Egr1, CDP had no effect on promoter activity in the context of primary bladder cancer cells (Figure 3D). The crucial event in the activation of NF- $\kappa \mathrm{B}$ is the phosphorylation of $\mathrm{I} \kappa \mathrm{B}$. In agreement with this, I $\mathrm{B}$ phosphorylation was induced $3 \mathrm{~h}$ after epidoxorubicin treatment, earlier than GRO- $\alpha$ expression (Figure 3E). The drug-induced GRO- $\alpha$ expression was significantly suppressed by either $\mathrm{I} \kappa \mathrm{B} \alpha$ super-repressor (a protein with mutations that render it resistant to stimulus-induced degradation) or $\mathrm{NF}-\kappa \mathrm{B}$ siRNA in PB2 cells (Figure 3F). c.

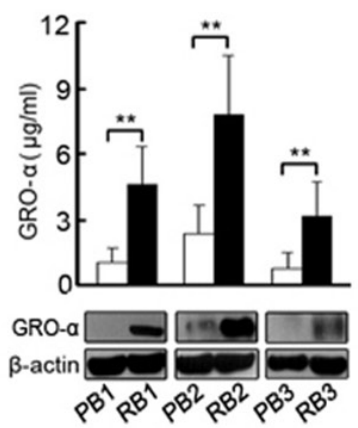

E.

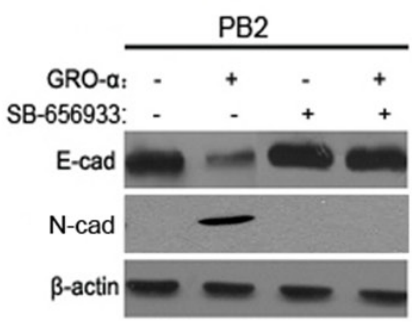

D.

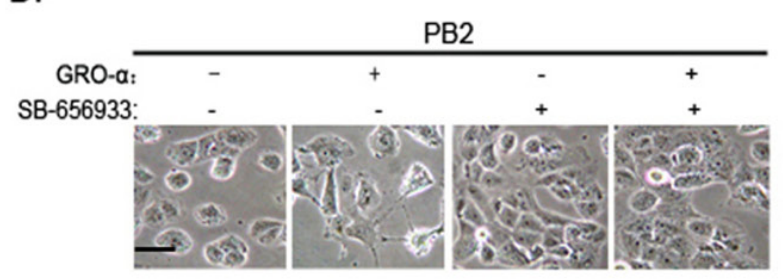

F.

G.

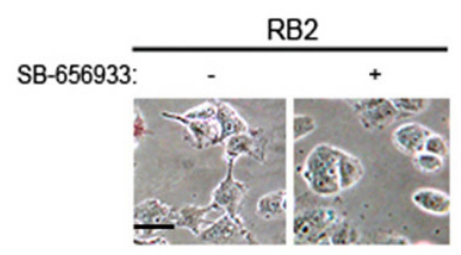

Figure 2: GRO- $\alpha$ derived from recurrent bladder cancer cells elicits epithelial-mesenchymal transition. (A) Cartoon depicting the experimental approach adopted to for the coculture Transwell system. (B) After PB1 cells were transwell-cultured with RB1 or stromal cells as described in (A), cell morphology and immunofluorescence of E-cadherin, F-actin were examined. (C) GRO- $\alpha$ secretion in supernatant, as well as its expression in cell lysates, in the recurrent cancer cells was evaluated by ELISA and western blot analysis,

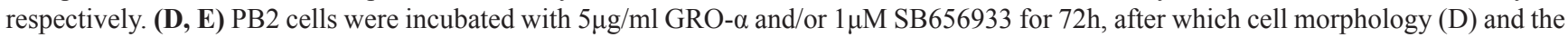
expression level of E-cadherin, N-cadherin (E) were examined. (F, G) RB2 cells were incubated with $1 \mu \mathrm{M}$ SB656933 for $72 \mathrm{~h}$, after which cell morphology (F) and the expression level of E-cadherin, N-cadherin (G) were examined. Experiments were performed three times independently, and results are shown as mean $\pm \mathrm{SD} .{ }^{* *}, \mathrm{p}<0.05$ versus the corresponding controls. Bars: $10 \mu \mathrm{m}$. 
Previous studies indicated that MAPK/p38 activation promotes cancer cell survival after chemotherapy $[11$, 12]. Epidoxorubicin induced p38 activation in $\mathrm{PB} 1$ cells at $\mathrm{G} 1 / \mathrm{S}$ phase but not at $\mathrm{G} 2 / \mathrm{M}$ phase (Figure $3 \mathrm{G}$ ). P38 signaling inhibition with SB202190 or SB203580 showed that $\mathrm{I} \kappa \mathrm{B}$ phosphorylation was p38 dependent, as p38 inhibition led to attenuation in drug-induced phospho-IкB abundance and subsequent GRO- $\alpha$ expression (Figure $3 \mathrm{H})$. These data suggest that epidoxorubicin induced GRO- $\alpha$ expression in primary bladder cancer cells at G1/S

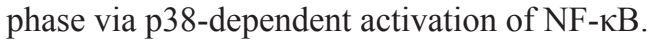

\section{GRO- $\alpha$-induced PI3K-mediated phosphorylation of Snail modulates Snail's subcellular localization and functions}

In order to identify which GRO- $\alpha$ response mediated EMT, we examined the effect of GRO- $\alpha$ or/and
SB656933 on expression of various transcription factors for EMT. In PB3 cells, snail expression was induced by GRO- $\alpha$ after $24 \mathrm{~h}$ treatment and significantly suppressed by pre-treatment of SB656933. However, the levels of other EMT-related molecules, such as slug, twist1, ZEB1 and SIP1, were not affected (Figure 4A). To explore the potential effect of GRO- $\alpha$ signaling upon the functions of Snail, we examined the impact of GRO- $\alpha$ on the ability of Snail to repress E-cadherin promoter activity. Our results showed that either GRO- $\alpha$ or constitutively active CXCR2 mutant had an inhibitory effect on E-cadherin promoter activity. GRO- $\alpha$-induced effects were largely abolished by knockdown of Snail (Figure 4B). We also examined the effect of GRO- $\alpha$ on two additional Snail-regulated genes, tight junction membrane protein occludin and estrogen synthetase enzyme aromatase. GRO- $\alpha$ treatment promoted the ability of Snail to repress transcription from both occludin- and aromatase-
A.

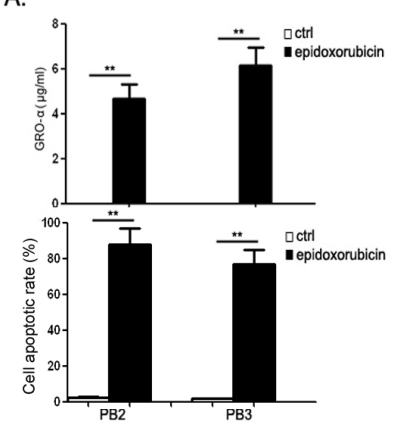

B.

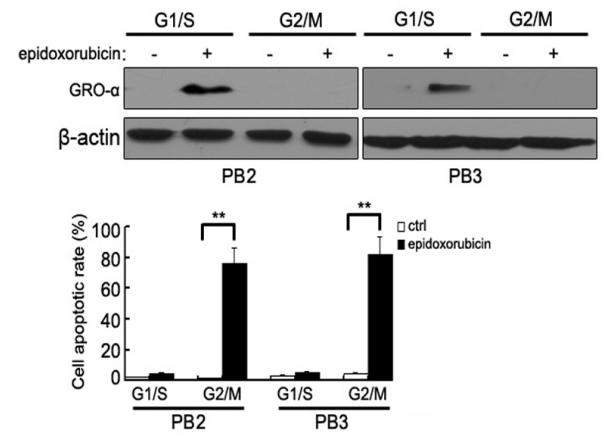

$\mathrm{F}$.

C.

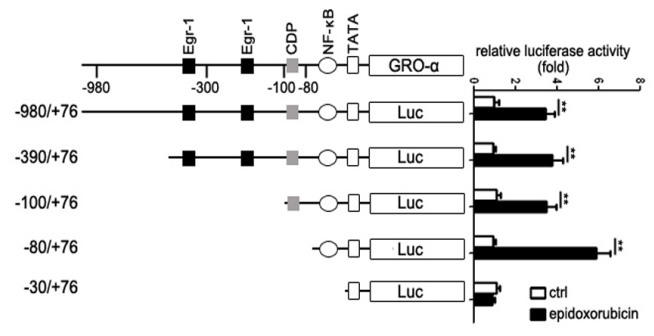

E.
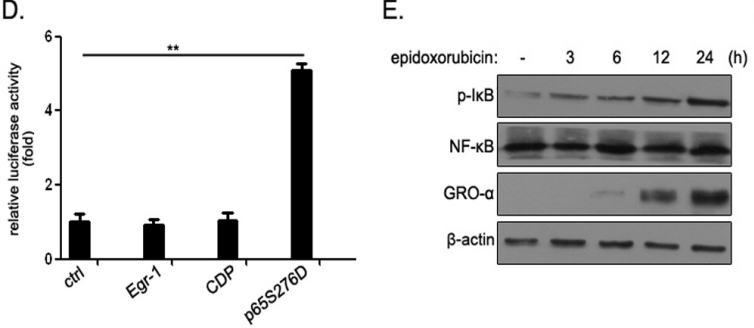

G.

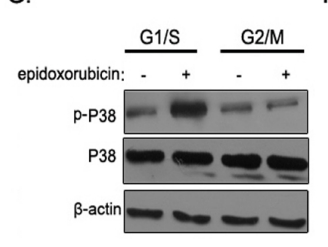

$\mathrm{H}$

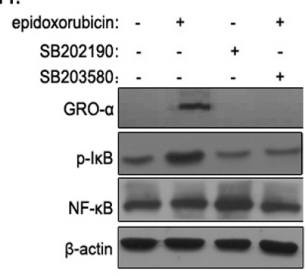

Figure 3: Epidoxorubicin induce GRO- $\alpha$ expression in bladder cancer cells in a cell cycle-dependent manner via the p38/MAPK-NF-кB pathway. (A) PB2 and PB3 cells were incubated with $1 \mu \mathrm{g} / \mathrm{ml}$ epidoxorubicin for $48 \mathrm{~h}$, after which GRO- $\alpha$ secretion and cell apoptotic rate were assessed by ELISA and flow cytometry, respectively. (B) PB2 and PB3 cells were synchronized in $\mathrm{G} 1 / \mathrm{S}$ boundary by the addition of $2 \mathrm{mmol} / 1$ hydroxyurea to the medium for $16 \mathrm{~h}$. G2/M synchronization was achieved by maintaining the cells inculture with $0.1 \mathrm{ng} / \mathrm{ml}$ nocodazole for $20 \mathrm{~h}$. After being synchronized to $\mathrm{G} 1 / \mathrm{S}$ or G2/M phase, cells were treated with $1 \mu \mathrm{g} / \mathrm{ml}$ epidoxorubicin for another $48 \mathrm{~h}$ to assess GRO- $\alpha$ expression or apoptotic rate. (C) PB2 cells were transiently transfected with $0.2 \mu \mathrm{g}$ of a series of 5'-deletion constructs of human GRO- $\alpha$ promoter reporter plasmids. Forty-eight hours later, the cells were either untreated or treated with $1 \mu \mathrm{g} / \mathrm{ml}$ epidoxorubicin for $8 \mathrm{~h}$, and luciferase activities were measured. (D) PB2 cells were transiently co-transfected with $0.2 \mu \mathrm{g}$ of pGRO- $\alpha$-Luc(-980/+76) and expression plasmids for Egr-1 (pCDNA3.1/Egr-1), CDP (pCDNA3.1/CDP) or p65S276D (pCDNA3.1/ p65S276D). After 48h, the cells were collected and assayed for luciferase activity. (E) PB2 cells were treated with $1 \mu \mathrm{g} / \mathrm{ml}$ epidoxorubicin for the indicated times, after which the expression level of phospho-IкB, NF- $\kappa$ B and GRO- $\alpha$ was determined. (F) PB2 cells were transiently

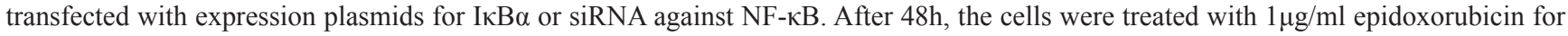
another $48 \mathrm{~h}$ and the expression level of GRO- $\alpha$ was examined. (G) After being synchronized to G1/S or G2/M phase as described in (B), PB1 cells were treated with $50 \mu \mathrm{g} / \mathrm{ml}$ epidoxorubicin for another $48 \mathrm{~h}$ to assess the expression level of phosphor-P38 and P38. (H) PB1 cells

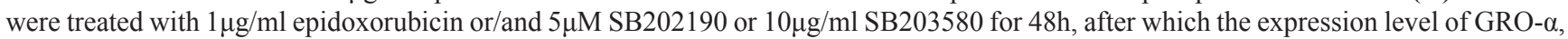

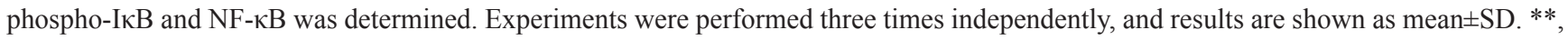
$\mathrm{p}<0.05$ versus the corresponding controls. 
promoter reporters. Consistent with the role of GRO- $\alpha$ in modulating Snail activity, inhibition of GRO- $\alpha-C X C R 2$ signaling with a dominant-negative CXCR2 relieved the repression effect of Snail on occludin and aromatase promoter activity (Figure 4C).

To gain deeper insights into the regulatory effect of GRO- $\alpha$-CXCR2 signaling on Snail functions, we examined the effect of GRO- $\alpha$ upon the subcellular localization of Snail in bladder cancer cells by scanning confocal microscopy. We found that knockdown of GRO- $\alpha$ expression by siRNA led to almost complete disappearance of nuclear Snail in RB3 cells, while GRO- $\alpha$ promoted nuclear translocation of cytoplasmic Snail in PB3 cells (Figure 5A). Interestingly, we noticed dose-dependent phosphorylation of Snail by GRO- $\alpha$ (Figure 5B). PI3K and p38 MAPK are two important downstream signaling pathways of CXCR2 [13-15]. Inhibition of PI3K pathway, but not p38 signaling, largely abolished GRO- $\alpha$-induced Snail phosphorylation in the RB3 cells (Figure 5C and Supplementary Figure 3A). To determine whether Snail was a novel substrate of PI3K, we then did the in vivo Snail phosphorylation assay. We cotransfected serum-starved 293 T cells with
HA-tagged Snail, with or without catalytically active PI3K (PI3KCA), after which the cells were labeled with [32P] orthophosphoric acid. It was found that HASnail could be phosphorylated by PI3KCA but not by wild type PI3K in serum-starved conditions (Figure 5D). To map the PI3K phosphorylation site in Snail, we identified five potential phosphorylation sites in Snail and substituted all potential serine or threonine residues with alanine in the potential phosphorylation sites by site-directed mutagenesis (Thr203Ala, Ser221Ala, Thr229Ala, Thr257Ala, and Ser246Ala) (Supplementary Figure $3 \mathrm{~B}$ ). The site of GRO- $\alpha$-induced PI3K-mediated phosphorylation on Snail was identified as Ser246 (Figure 5E). Next, we compared the repression activity of Snail Ser246Ala mutant with that of wildtype Snail against a Snail-target gene reporter assay. GRO- $\alpha$-mediated repression of transcription from the E-cadherin, occludin and aromatase promoters were significantly relieved by the mutant Snail Ser246Ala (Figure 5F). These results suggest that GRO- $\alpha$-induced phosphorylation of Snail modulated its transcriptional activity by increasing accumulation of Snail in the nucleus.

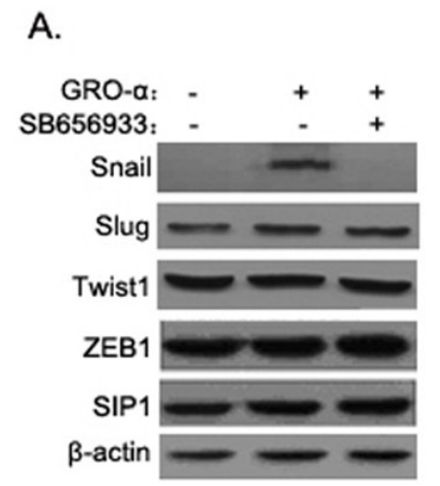

B.

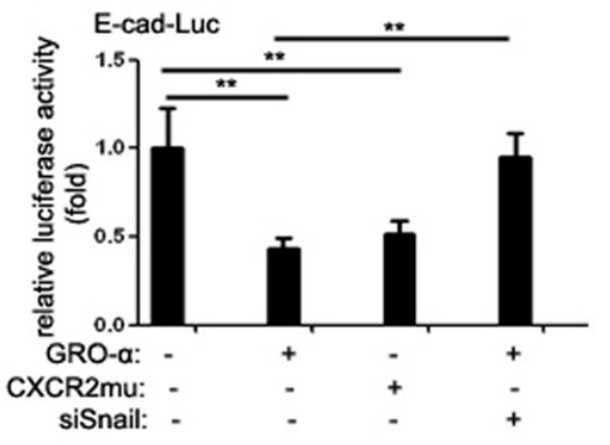

C.

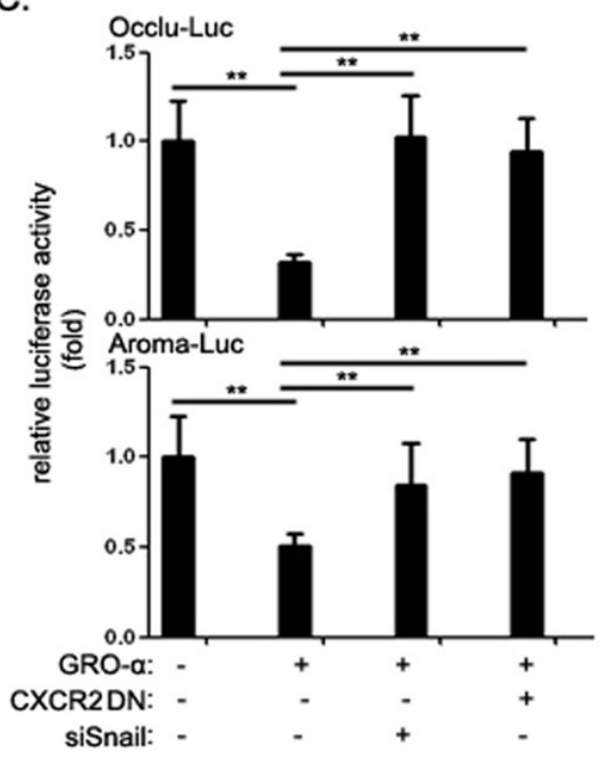

Figure 4: The GRO- $\alpha-C X C R 2$ axis promotes Snail expression and its repressor activity. (A) PB2 cells were treated with $1 \mu \mathrm{g} /$ $\mathrm{ml}$ epidoxorubicin or/and $1 \mu \mathrm{M} \mathrm{SB} 656933$ for 24h, after which the expression level of Snail, Slug, Twist1, ZEB1 and SIP1 was assessed by western blot analysis. (B) E-cadherin luciferase reporter assay: 100ng of luciferase reporter constructs carrying human E-cadherin promoter were transfected into PB2 cells singly or together with CXCR2mu expression vector or cotransfection of Snail siRNA, respectively; After treatment with or without $5 \mu \mathrm{g} / \mathrm{ml} \mathrm{GRO}-\alpha$ for $24 \mathrm{~h}$, the cells were collected and assayed for luciferase activity. (C) Occludin and aromatase luciferase reporter assay: 100ng of luciferase reporter constructs carrying human occludin and aromatase promoter were transfected into PB2 cells singly or together with CXCR2DN expression vector or cotransfection of Snail siRNA, respectively; After treatment with or without $5 \mu \mathrm{g} / \mathrm{ml}$ GRO- $\alpha$ for $24 \mathrm{~h}$, the cells were collected and assayed for luciferase activity. Experiments were performed three times independently, and results are shown as mean $\pm \mathrm{SD} .{ }^{* *}, \mathrm{p}<0.05$ versus the corresponding controls. 


\section{Overcoming post-therapeutic recurrence of bladder cancers by disrupting GRO- $\alpha$-mediated EMT}

Due to the significance of EMT in cancer progression, we tested whether abrogating the process of EMT would prevent post-therapeutic recurrence. We generated the PB2 cells expressing GRO- $\alpha$ shRNA under the conditional control of Tet (Figure 6A), after which these cells were subcutaneously transplanted into NOG mice. All 8-weekold mice harboring non-invasive bladder cancer cells were subjected to epidoxorubicin alone, or Tet alone, or epidoxorubicin plus Tet treatment. Treatment with epidoxorubicin alone produced $>82 \%$ tumor regression within 4 weeks (Figure 6B), but significantly increased the percentage of N-cad+ cells, accompanied by reduced quantity of E-cad+ cells, in residual lesions (Figure 6D). Recurrence was observed in 9 out of 10 such mice within 2 weeks of treatment cessation (Figure 6B). In stark contrast, blocking GRO- $\alpha$ production by administration of Tet significantly abolished the process of EMT (Figure 6C and 6D). Treatment with epidoxorubicin plus Tet effectively decreased tumor size, and no recurrence was noted in any subject (Figure 6B).

\section{DISCUSSION}

Bladder cancer carries a high risk of recurrence and poor prognosis due to muscle invasion and metastasis. In this study, we observed that bladder cancer recurrence was associated with the aberrant mesenchymal phenotype. Mesenchymal recurrent bladder cancer cells secreted multiple cytokines and growth factors, including transforming growth factor beta, interleukin-8, EGF, Wnt, and granulocyte-macrophage colony-stimulating factor, many of which are involved in malignancy progression in various types of carcinomas [16-18]. In particular, mesenchymal recurrent $\mathrm{BCa}$ cells undergoing chemotherapy are the major source of GRO- $\alpha$. For cells at G1/S phase, chemotherapy drugs induced the expression of GRO- $\alpha$. This was not observed in cells at G2/M phase. Our data well explain how bladder cancer cells respond to chemotherapy heterogeneously in terms of cell cycle phases. GRO- $\alpha$ is a member of the CXC chemokine family. The biological function of GRO- $\alpha$ is primarily mediated via CXCR2, a seven-transmembrane G proteincoupled receptor. GRO- $\alpha$ has been reported to play a critical role in tumorigenesis, angiogenesis, and metastasis $[13-15,19,20]$. Although a higher expression of GRO- $\alpha$
A.

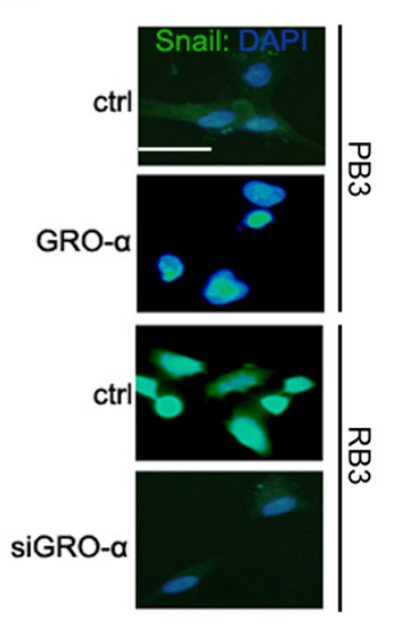

B.

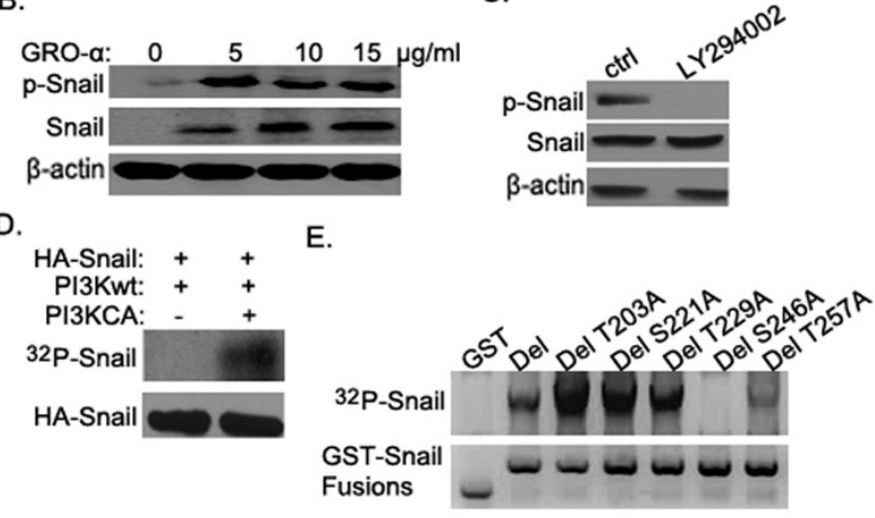

F.

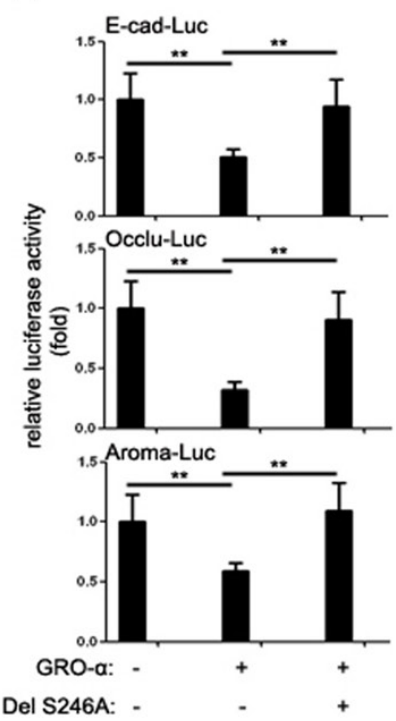

Figure 5: GRO- $\alpha$ regulation of Snail phosphorylation and subcellular localization. (A) Confocal microscopic analysis of Snail localization in RB3 cells transfected with GRO- $\alpha$ siRNA or inPB3 cells treated with $5 \mu \mathrm{g} / \mathrm{ml}$ GRO- $\alpha$ for $36 \mathrm{~h}$. (B) PB3 cells were treated with the indicated concentrations of GRO- $\alpha$ for $36 \mathrm{~h}$, after which the expression level of phospho-Snail and Snail were assessed. (C) RB3 cells were treated with 50 $\mathrm{gg} / \mathrm{ml} \mathrm{LY294002} \mathrm{for} \mathrm{48h,} \mathrm{after} \mathrm{which} \mathrm{the} \mathrm{expression} \mathrm{level} \mathrm{of} \mathrm{phospho-Snail} \mathrm{and} \mathrm{Snail} \mathrm{were} \mathrm{assessed.} \mathrm{(D)} 293$ $\mathrm{T}$ cells were serum-starved for 1 day and then transfected with Snail expression vector together with either wild-type PI3K or constitutively active form of PI3K (PI3KCA). Cells were labeled with [32P]orthophosphoric acid and cell lysates were immunoprecipitated with an anti-HA mAb. Phosphorylation was visualized by autoradiography with a PhosphoImager. (E) In vitro phosphorylation of Snail deletion constructs by GRO- $\alpha$. All five different point mutation constructs were made from a Snail deletion construct (amino acids 181-264). (F) E-cadherin, occludin and aromatase luciferase reporter assay: 100ng of luciferase reporter constructs carrying human E-cadherin, occludin and aromatase promoter were transfected into PB3 cells singly or together with Snail DelS246A expression vector; After treatment with or without $5 \mu \mathrm{g} / \mathrm{ml} \mathrm{GRO}-\alpha$ for $24 \mathrm{~h}$, the cells were collected and assayed for luciferase activity. Experiments were performed three times independently, and results are shown as mean $\pm \mathrm{SD}$. **, $\mathrm{p}<0.05$ versus the corresponding controls. Bar: $5 \mu \mathrm{m}$. 
was associated with the invasive phenotype of bladder cancer both in vitro and in vivo [21, 22], the detailed mechanism of this chemokine in invasive potential of bladder cancer is poorly understood. As the GRO- $\alpha$ receptor CXCR2 is expressed in all bladder cancer cells irrespective of the mesenchymal phenotype, it is probable that secretion of GRO- $\alpha$ is associated with bladder cancer recurrence via an autocrine loop involving its receptor. Here, we demonstrated that the interaction between GRO- $\alpha$ and CXCR2 induces activation of PI3K signaling. GRO- $\alpha$-induced PI3K-mediated phosphorylation of Snail on Ser246 promotes Snail's nuclear accumulation and consequently its repressor activity on the promoter of
E-cadherin. Ultimately, the bladder cancer cells undergo EMT and become highly invasive. In line with these, our in vivo studies demonstrated that interfering with the GRO- $\alpha /$ CXCR2 axis considerably disrupted the process of EMT, suggesting that the GRO- $\alpha / \mathrm{CXCR} 2$ axis represents the driving force of mesenchymal transformation. Mounting evidence has implied the contributions of EMT in the emergence of treatment failure and tumor recurrence. Accordingly, abolishing GRO- $\alpha$-induced EMT successfully prevented post-chemotherapeutic relapse. In this sense, disrupting the GRO- $\alpha$-CXCR2 axis in NMIBC may represent a promising alternative to prevent postchemotherapy cancer progression and recurrence.

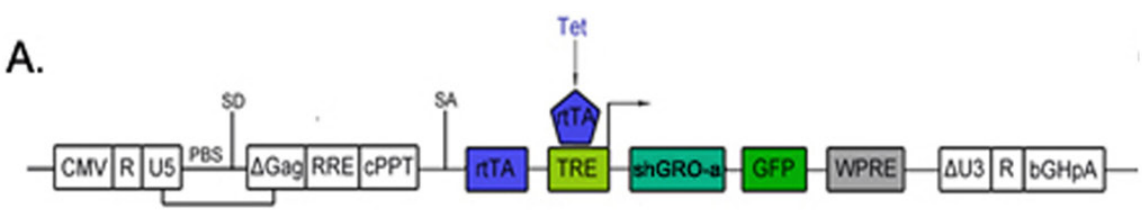

B.

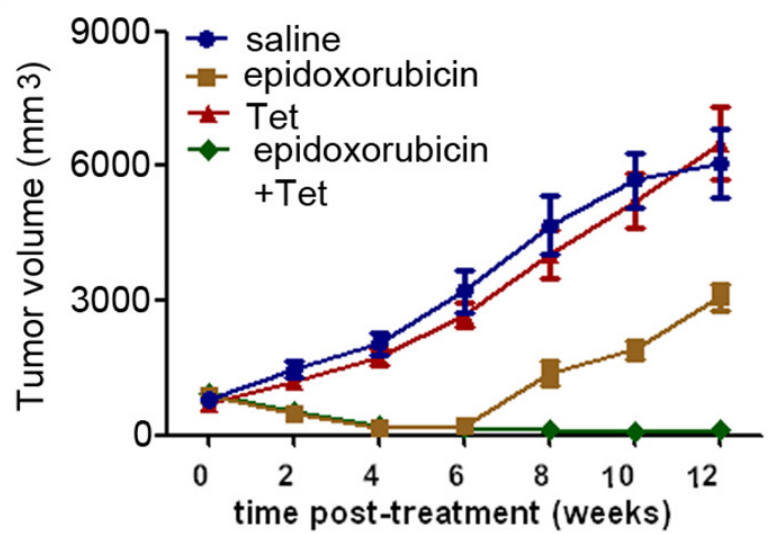

C.

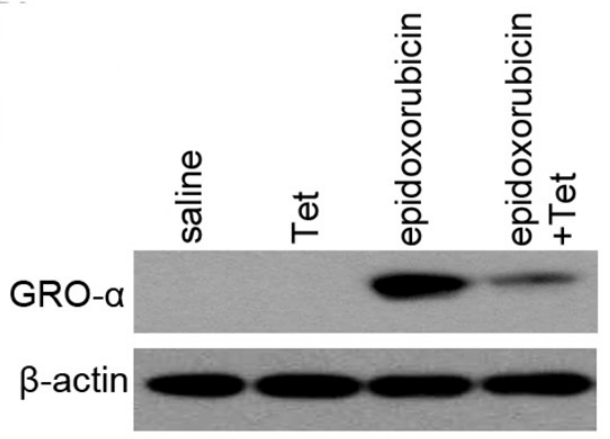

D.

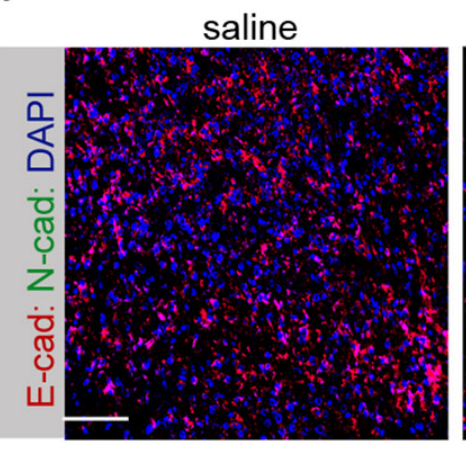

Tet

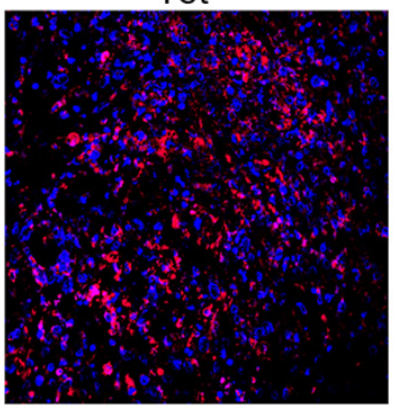

epidoxorubicin

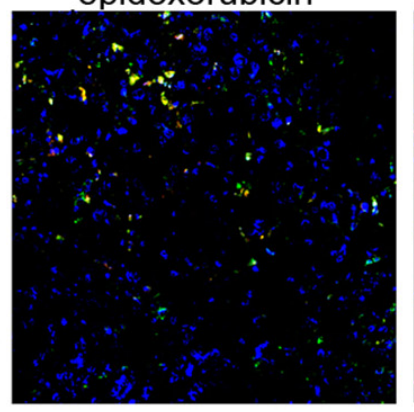

epidoxorubicin+Tet

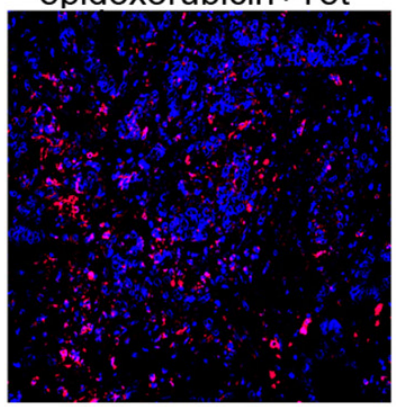

Figure 6: Interrupting the GRO- $\alpha-C X C R 2$ axis prevents EMT and abolishes post-therapeutic recurrence in bladder cancers. (A) Schematic representation of the Tet-on lentiviral vector used in this study. PB2 cells transfected with the lentiviral vector in (A) were subcutaneously implanted into NOG mice and allowed to form obvious tumors in 4 weeks. Mice were randomized into groups $(\mathrm{n}=15 \mathrm{each})$ that received saline, epidoxorubicin $(0.04 \mathrm{mg} / \mathrm{kg})$, Tet $(4 \mathrm{mg} / \mathrm{kg})$ or epidoxorubicin+Tet. All treatments were repeated twice weekly for four weeks. (B) Mice were monitored weekly for tumor volume at the indicated times. (C) After cessation of the treatments, the expression level of GRO- $\alpha$ in residual tumors of each group was assessed. (D) Immunofluorescence of E-cadherin and N-cadherin in residual tumors of each group after cessation of the treatments. Bars: $50 \mu \mathrm{m}$. 


\section{MATERIALS AND METHODS}

\section{Ethics statement}

This study was approved by the institutional review board of Changzheng Hospital of the Second Military Medical University (Shanghai, China) and written informed consent was obtained from all patients who provided bladder cancer tissues.

\section{Cell culture and reagents}

Tissues were harvested from bladder specimens removed at the time of Transurethral resection of bladder tumor (TURBT) for primary and recurrent carcinoma of the bladder in the same patients. Cells were cultured at $37^{\circ} \mathrm{C}$ with $5 \% \mathrm{CO} 2$ in RPMI 1640 supplemented with $10 \% \mathrm{v} / \mathrm{v}$ Fetal bovine serum (FBS) and penicillin(100 units $/ \mathrm{ml}) /$ streptomycin $(0.1 \mathrm{mg} / \mathrm{ml})$. Recurrent bladder tumor cultures from three patients were sorted as cancer cells and stromal cells by CK7 antibody via FACS LSRII (BD Biosciences). Cancer cells were termed as PB1, PB2 and $\mathrm{PB} 3$ in primary cancers, and RB1, RB2 and RB3 in recurrent cancers. $293 \mathrm{~T}$ cells were culture at $37^{\circ} \mathrm{C}$ with $5 \% \mathrm{CO} 2$ in RPMI 1640 supplemented with $10 \% \mathrm{v} / \mathrm{v}$ FBS and penicillin (100units/ml)/streptomycin $(0.1 \mathrm{mg} / \mathrm{ml})$. SB656933, SB202190, SB203580 and LY294002 were purchased from Calbiochem (San Diego, CA, USA) and Human recombinant GRO- $\alpha$ was from ProSpec Biology (East Brunswich, NJ, USA). The firefly and Renilla DualGlo $^{\mathrm{TM}}$ Luciferase Assay System was purchased from Promega (Madison, WI, USA). The pRL-null plasmid, which encodes Renilla luciferase, was purchased from Promega.

\section{Xenograft studies}

Subcutaneous xenograft tumor models were established based on 4-week-old female BALB/c-nu mice. The mice were housed in a pathogen-free facility at the Second Military Medical University. All animal protocols are in compliance with ethical regulations and have been approved by the Institutional Animal Care Use Committee of Second Military Medical University. For Sub-Q xenografts, the indicated cells suspended in $100 \mu$ l Hanks buffered saline solution were injected subcutaneously in the axillary region of the right chest in BALB/c-nu/nu mice. Tumor volume was measured every 2 days starting from day 4 post-injection to monitor the extent of tumor growth, regression and recurrence. Tumor volume was calculated using the formula: $1 / 2$ (Length $\times$ Width2). When tumor reached $\sim 1 \mathrm{~cm}$ in diameter, mice were randomly divided into the indicated groups and the mean tumor volumes of each group were similar before the treatment started. No mice were excluded after the treatment started. To determine the effects of epidoxorubicin treatment alone or epidoxorubicin plus tetracycline (Tet) treatment, tumor-bearing mice were treated with intraperitoneal (i.p.) epidoxorubicin $(0.04 \mathrm{mg} / \mathrm{kg}$; Sigma-Aldrich, Dorset, UK) or/and Tet (4mg/kg; Sigma-Aldrich, Dorset, UK), twice weekly for 4 weeks.

\section{Immunofluorescence}

Cells were washed with PBS twice, fixed with 4\% formaldehyde for $15 \mathrm{~min}$, permeabilized with $0.1 \%$ Triton $\mathrm{X}-100 / \mathrm{PBS}$ for $5 \mathrm{~min}$, and blocked by $3 \%$ bovine serum albumin for $30 \mathrm{~min}$ at room temperature. The coverslips were stained with primary antibodies overnight at $4^{\circ} \mathrm{C}$ followed by incubation with secondary antibody for 30 min at $37^{\circ} \mathrm{C}$. Cells were washed three times with PBS in a dark chamber. The coverslips were washed as described above, inverted, mounted on slides using DAPI (Life Technologies), and examined under a Leica confocal microscope.

\section{Western blot analysis}

Protein preparations from the indicated cells were obtained by lysing samples in $50 \mathrm{mmol} / \mathrm{L}$ of TRIS $(\mathrm{pH}$ 7.5), $100 \mathrm{mmol} / \mathrm{L}$ of $\mathrm{NaCl}, 1 \% \mathrm{NP} 40,0.1 \%$ Triton, 2 $\mathrm{mmol} / \mathrm{L}$ of EDTA, $10 \mathrm{Ag} / \mathrm{mL}$ of aprotinin, and $100 \mathrm{Ag} / \mathrm{mL}$ of phenylmethylsulfonyl-fluoride. Prestained molecular weight standards were from Bio-Rad (Milan). Proteins separated on the polyacrylamide gels were blotted on a PVDF membrane. The membrane was stained with Ponceau S (Sigma) to enable us to evaluate the success of transfer, and to locate the molecular weight markers. Free protein binding sites on the PVDF membrane were blocked with nonfat dry milk and a Tween 20/TBS solution. The membranes were washed and stained with specific primary antibodies and with secondary antisera, and then conjugated with horseradish peroxidase (SigmaAldrich) diluted 1:2,000. The anti-E-cadherin, anti-Ncadherin, anti-CXCR2, anti-phospho-P38, anti-P38,

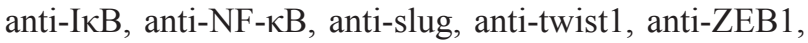
anti-Snail and anti-SIP1 antibodies were purchased from Abcam, and the anti-GRO- $\alpha$ antibody was from Santa Cruz. The luminescent signal was visualized with the ECL Western blotting detection reagent kit (Amersham) and quantified by scanning with a Discover Pharmacia scanner equipped with a Sun Spark Classic Workstation.

\section{Isolation of primary epithelial and stromal cells from bladder specimens}

Tumor specimens were processed within 1 to $2 \mathrm{~h}$ of surgery. Each sample was minced in small fragments $(<1 \mathrm{~mm} 3)$, in a sterile environment. Tissue fragment were then digested at $37^{\circ} \mathrm{C}$, for 2 to $4 \mathrm{~h}$ in a solution of type IV collagenase, $1 \mathrm{mg} / \mathrm{ml}$ (Sigma-Aldrich), containing $40 \mathrm{mg} / \mathrm{ml}$ of bovine serum albumin, $2 \mathrm{mg} / \mathrm{ml}$ of glucose, 
100 units/ml of penicillin and $100 \mathrm{mg} / \mathrm{ml}$ of streptomycin (P+S; Sigma-Aldrich), $50 \mathrm{mg} / \mathrm{L}$ of gentamicin (SigmaAldrich), and $1.25 \mathrm{mg} / \mathrm{L}$ of Fungizone (Life Technologies). After incubation, the samples were extensively rinsed with PBS and suspended in culture media. Each sample was divided into two aliquots, one to be frozen and one to be cultured. Cells underwent three cycles of centrifugation and were differentially separated by centrifugation. Briefly, increasing speeds of centrifugation (40-100-200×g) generated three cell populations: epithelial-enriched (EE), stromal-enriched (SE), and organoid substance (OS). The cells were seeded separately in 24-wells at a density of 105 cells/well and cultured in standard medium: RPMI 1640 supplemented with $2 \mathrm{mmol} / \mathrm{L}$ of glutamine (SigmaAldrich), P+S, $15 \mathrm{mmol} / \mathrm{L}$ HEPES (Sigma-Aldrich), and with different percentages of FBS and hormones and growth factors. EE and OS cells were cultured in $0.5 \%$ FBS and a mixture of six supplements $(6 \mathrm{H})$ for at least 15 days. SE were cultured in 10\% FBS medium. Because after 15 days, the growth rate of EE cells was very low or null, we enzymatically [trypsin-EDTA solution (Cambrex) for $2 \mathrm{~min}$ at $37^{\circ} \mathrm{C}$ ] detached and seeded the cells on top of the sister semiconfluent SE dish. Cells were maintained at $37^{\circ} \mathrm{C}$ in a humidified atmosphere of $5 \% \mathrm{CO} 2$. The medium was renewed twice weekly. The two cell types were easily identified by their morphology.

\section{Transwell cell invasion and coculture}

For cell invasion assay, Transwell chamber (Corning Inc.) was coated with Matrigel and DMEM (v/v 1:8), with cultures below $80 \%$ confluence to ensure proper starvation of cells without becoming confluent during the 24starvation period. The cell seeding concentration $(5 \times 105$ cells/ml) was diluted with $150 \mu \mathrm{l}$ serum-free medium and added to Matrigel mixture. $500 \mu \mathrm{l}$ DMEM with $10 \%$ FBS was added to a lower compartment. Cultures were incubated for $36 \mathrm{~h}$. The invaded cells were stained and counted according to the manufacturers' instructions. For cell coculture assay, an equal number of PB1 cells and RB1 cells were seeded in the upper compartment and lower compartment of Transwell chamber, respectively. Cell supernatants were collected for further assays.

\section{Oncosphere formation assay}

For oncosphere formation assay, cells were trypsinized, counted as $5 \times 10^{5} / \mathrm{ml}$, mixed in FBS-free RPMI 1640 medium containing 20ng/ml EGF (SigmaAldrich), 10ng/ml FGF2 (Sigma-Aldrich), B27 (GIBCO), and ITSS (Roche), plated onto ultralow attachment plates (Corning), and incubated at $37^{\circ} \mathrm{C}$ with $5 \% \mathrm{CO}_{2}$. Fresh growth medium was added every $48 \mathrm{~h}$. To determine the frequency of oncosphere-forming cells, one thousand tumor cells were distributed into each well in lowattachment 24-well tissue culture plates in the stem cell medium. The number of oncospheres in each well was counted on day 7 . At this cell density $(1,000$ cells per well) in the 24-well plates, cells did not cluster together. Oncosphere formation was determined at the indicated time points by automated imaging on an inverted microscope (Axiovert 100M, Carl Zeiss).

\section{CCK-8 cell proliferation}

Cells were counted and the concentration of the cell suspension was adjusted to $5 \times 10^{4}$ cells $/ \mathrm{ml}$. Using serial dilution, cell suspension $(100 \mu \mathrm{l})$ was added to each well of the 96-well microplate. $10 \mu \mathrm{l}$ cell counting Kit-8 (CCK-8) was added to each well and reacted in a $\mathrm{CO} 2$ incubator for $4 \mathrm{~h}$. Absorbance was measured at $450 \mathrm{~nm}$ on a microplate reader.

\section{Flow cytometry}

For cell apoptosis and cell cycle, $1 \times 10^{5}$ cells were seeded per well in a 12 -well plate for $24 \mathrm{~h}$ and then treated with $10 \mu \mathrm{mol} / \mathrm{L}$ epidoxorubicin. After $24 \mathrm{~h}$ incubation, cells were collected, stained with propidium iodide (PI) and APC-conjugated Annexin V (Apoptosis detection kit, Invitrogen), and analyzed by flow cytometry and software (BD Biosciences).

\section{In vivo phosphorylation}

293 T cells were transfected with either Snail expression vector or the construct with the indicated point mutations and cotransfected with with or without catalytically active PI3K (PI3KCA). Cells were labeled with [32P] orthophosphoric acid overnight. Twenty-four hours after transfection, cell lysates were immunoprecipitated with an anti-HA monoclonal antibody $(\mathrm{mAb})$ and then separated by $10 \%$ SDS-PAGE. Labeling was visualized by autoradiography with a PhosphorImager.

\section{Luciferase reporter assays}

Cells in 24-well plates were transiently transfected with the indicated promoter reporter constructs and pRLTK Renilla luciferase plasmid (Promega) using Fugene 6 (Roche). Cell extracts were prepared after transfection, and luciferase activity was measured using the Dual-Luciferase Reporter Assay System (Promega).

\section{Protein quantitation and identification}

The supernatant samples from PB1 and RB1 were in-gel digested enzymatically and analyzed by LC-MS/ MS. The database search was performed using both the X!Tandem and SEQUEST algorithms. Quantification was first performed using Mascot 2.2. Using the Mascot quantification method, protein quantification was 
only performed on proteins identified by two or more peptides with scores above the identity threshold. Protein differential expression was also assessed at the peptide level. All peptides were used to calculate global mean and S.D. of peptide ratios (47-1/D3). The differentially expressed peptides were used to infer differentially expressed proteins.

\section{Statistical analysis}

All statistical analyses were performed with SPSS software (SPSS, Chicago, IL). Analysis of variance, Student $\mathrm{t}$ test and Dunnett's multiple comparison test were used to compare mean values. Data are presented as mean \pm SD. A $p$ value of $<.05$ was defined as statistically significant.

\section{ACKNOWLEDGMENTS AND FUNDING}

This work is supported by grants from Shanghai Municipal Commission of Health and Family Planning (No. 201440511); the National Natural Science Foundation of China for Youths (No. 81602238, No. 81001136); the National Natural Science Foundation of China (No. 81170637); Shanghai Committee of Science and Technology General Program for Medicine (No. 11JC1402302); the Military Fund for Health Care (CWS13BJ09); the Youth Talent Sailing Program of Shanghai Science and Technology Committee (17YF1425400); the Youth Foundation of Second Military Medical University (2016QN17); and the Key Project of Science and Innovation Foundation of Shanghai Ministry of Education (14zz084).

\section{CONFLICTS OF INTEREST}

The authors declare no competing financial interests.

\section{REFERENCES}

1. Anastasiadis A, de Reijke TM. Best practice in the treatment of nonmuscle invasive bladder cancer. Ther Adv Urol. 2012; 4:13-32.

2. Babjuk M, Burger M, Zigeuner R, Shariat SF, van Rhijn BW, Compérat E, Sylvester RJ, Kaasinen E, Böhle A, Palou Redorta J, Rouprêt M, and European Association of Urology. EAU guidelines on non-muscle-invasive urothelial carcinoma of the bladder: update 2013. Eur Urol. 2013; 64:639-53.

3. Lammers RJ, Palou J, Witjes WP, Janzing-Pastors MH, Caris CT, Witjes JA. Comparison of expected treatment outcomes, obtained using risk models and international guidelines, with observed treatment outcomes in a Dutch cohort of patients with non-muscle-invasive bladder cancer treated with intravesical chemotherapy. BJU Int. 2014; 114:193-201.
4. Yang J, Weinberg RA. Epithelial-mesenchymal transition: at the crossroads of development and tumor metastasis. Dev Cell. 2008; 14:818-29.

5. Thiery JP. Epithelial-mesenchymal transitions in tumour progression. Nat Rev Cancer. 2002; 2:442-54.

6. Creighton CJ, Gibbons DL, Kurie JM. The role of epithelial-mesenchymal transition programming in invasion and metastasis: a clinical perspective. Cancer Manag Res. 2013; 5:187-95.

7. Kang Y, Massagué J. Epithelial-mesenchymal transitions: twist in development and metastasis. Cell. 2004; 118:277-79.

8. Baumgart E, Cohen MS, Silva Neto B, Jacobs MA, Wotkowicz C, Rieger-Christ KM, Biolo A, Zeheb R, Loda M, Libertino JA, Summerhayes IC. Identification and prognostic significance of an epithelial-mesenchymal transition expression profile in human bladder tumors. Clin Cancer Res. 2007; 13:1685-94.

9. Li QQ, Xu JD, Wang WJ, Cao XX, Chen Q, Tang F, Chen ZQ, Liu XP, Xu ZD. Twist1-mediated adriamycin-induced epithelial-mesenchymal transition relates to multidrug resistance and invasive potential in breast cancer cells. Clin Cancer Res. 2009; 15:2657-65.

10. Kajiyama H, Shibata K, Terauchi M, Yamashita M, Ino K, Nawa A, Kikkawa F. Chemoresistance to paclitaxel induces epithelial-mesenchymal transition and enhances metastatic potential for epithelial ovarian carcinoma cells. Int J Oncol. 2007; 31:277-83.

11. Guo X, Ma N, Wang J, Song J, Bu X, Cheng Y, Sun K, Xiong H, Jiang G, Zhang B, Wu M, Wei L. Increased p38MAPK is responsible for chemotherapy resistance in human gastric cancer cells. BMC Cancer. 2008; 8:375.

12. Villedieu M, Deslandes E, Duval M, Héron JF, Gauduchon P, Poulain L. Acquisition of chemoresistance following discontinuous exposures to cisplatin is associated in ovarian carcinoma cells with progressive alteration of FAK, ERK and $\mathrm{p} 38$ activation in response to treatment. Gynecol Oncol. 2006; 101:507-19.

13. Acharyya S, Oskarsson T, Vanharanta S, Malladi S, Kim J, Morris PG, Manova-Todorova K, Leversha M, Hogg N, Seshan VE, Norton L, Brogi E, Massagué J. A CXCL1 paracrine network links cancer chemoresistance and metastasis. Cell. 2012; 150:165-78.

14. Benelli R, Stigliani S, Minghelli S, Carlone S, Ferrari N. Impact of CXCL1 overexpression on growth and invasion of prostate cancer cell. Prostate. 2013; 73:941-51.

15. Lv M, Xu Y, Tang R, Ren J, Shen S, Chen Y, Liu B, Hou Y, Wang T. miR141-CXCL1-CXCR2 signaling-induced Treg recruitment regulates metastases and survival of non-small cell lung cancer. Mol Cancer Ther. 2014; 13:3152-62.

16. Gao D, Vahdat LT, Wong S, Chang JC, Mittal V. Microenvironmental regulation of epithelial-mesenchymal transitions in cancer. Cancer Res. 2012; 72:4883-89. 
17. Lamouille S, Xu J, Derynck R. Molecular mechanisms of epithelial-mesenchymal transition. Nat Rev Mol Cell Biol. 2014; 15:178-96.

18. Thiery JP, Acloque H, Huang RY, Nieto MA. Epithelialmesenchymal transitions in development and disease. Cell. 2009; 139:871-90.

19. Sharma B, Nawandar DM, Nannuru KC, Varney ML, Singh RK. Targeting CXCR2 enhances chemotherapeutic response, inhibits mammary tumor growth, angiogenesis, and lung metastasis. Mol Cancer Ther. 2013; 12:799-808.

20. Wang B, Hendricks DT, Wamunyokoli F, Parker MI. A growth-related oncogene/CXC chemokine receptor
2 autocrine loop contributes to cellular proliferation in esophageal cancer. Cancer Res. 2006; 66:3071-77.

21. Miyake M, Lawton A, Goodison S, Urquidi V, GomesGiacoia E, Zhang G, Ross S, Kim J, Rosser CJ. Chemokine (C-X-C) ligand 1 (CXCL1) protein expression is increased in aggressive bladder cancers. BMC Cancer. $2013 ; 13: 322$.

22. Kawanishi H, Matsui Y, Ito M, Watanabe J, Takahashi T, Nishizawa K, Nishiyama H, Kamoto T, Mikami Y, Tanaka Y, Jung G, Akiyama H, Nobumasa H, et al. Secreted CXCL1 is a potential mediator and marker of the tumor invasion of bladder cancer. Clin Cancer Res. 2008; 14:2579-87. 